\title{
Population Dynamics for Renewables in Electricity Markets: A Minority Game View
}

\author{
Papakonstantinou, Athanasios; Pinson, Pierre
}

Published in:

Proceedings of 2016 International Conference on Probabilistic Methods Applied to Power Systems

Link to article, DOI:

10.1109/PMAPS.2016.7764068

Publication date:

2016

Document Version

Peer reviewed version

Link back to DTU Orbit

Citation (APA):

Papakonstantinou, A., \& Pinson, P. (2016). Population Dynamics for Renewables in Electricity Markets: A Minority Game View. In Proceedings of 2016 International Conference on Probabilistic Methods Applied to Power Systems IEEE. https://doi.org/10.1109/PMAPS.2016.7764068

\section{General rights}

Copyright and moral rights for the publications made accessible in the public portal are retained by the authors and/or other copyright owners and it is a condition of accessing publications that users recognise and abide by the legal requirements associated with these rights.

- Users may download and print one copy of any publication from the public portal for the purpose of private study or research.

- You may not further distribute the material or use it for any profit-making activity or commercial gain

- You may freely distribute the URL identifying the publication in the public portal 


\title{
Population Dynamics for Renewables in Electricity Markets: A Minority Game View
}

\author{
Athanasios Papakonstantinou and Pierre Pinson \\ Dept. of Electrical Engineering \\ Technical University of Denmark \\ Kgs. Lyngby 2800 \\ athpapa@elektro.dtu.dk ppin@elektro.dtu.dk
}

\begin{abstract}
The dominance of fluctuating and intermittent stochastic renewable energy sources (RES) has introduced uncertainty in power systems which in turn, has challenged how electricity market operate. In this context, there has been significant research in developing strategies for RES producers, which however typically focuses on the decision process of a single producer, assuming unrealistic access to aspects of information about the power system. This paper analyzes the behavior of an entire population of stochastic producers in an electricity market using as basis a minority game: the El Farol Bar problem. We illustrate how uncomplicated strategies based on a adaptive learning rules lead to the coordination among RES producers and a Pareto efficient outcome.
\end{abstract}

\section{INTRODUCTION}

The deployment of stochastic renewable energy sources (RES) e.g. wind and solar power, has been steadily increasing, bringing significant economic and environmental benefits. As renewable generation approaches grid parity, stochastic producers are asked to participate in electricity markets under the rules applied to conventional producers. Although now they may face regulation costs due to imbalances between their dayahead market offers and real-time production, they can also employ trading strategies in the day-ahead markets that may offset the cost of imbalances.

There has been an increasing scientific interest in addressing such issues. Specifically, [1] identify the optimal offer for a price-taker wind power producer highlighting the benefits of probabilistic offers against point forecasts. However, the assumption of the producer being a price-taker does not hold in zonal markets dominated by renewables, e.g., the Western Danish price area of Nord Pool's 'elspot' [2]. Recent works modeled the participation of a price-maker wind power producer in day-ahead [3] and real-time [4] markets, as well as in a joint day-ahead and real-time stochastic market [5].

Although the aforementioned approaches bring significant contributions, they have limitations. First, the stochastic producers rely on knowledge that is unlikely to be available to them, such as complete information of the power system's attributes and access to the predictive distributions of all other stochastic producers, while assuming that they accurately model real-time production. Second, they assume that only a single producer is strategic and capable of devising an optimal strategy, with the rest following without a reaction. That is, they do not capture the inherent dynamics of participating in a market, with the exception being the formulation of the competition among stochastic producers as an equilibrium program with equilibrium constraint [6]. However, such techniques are computationally demanding with reduced tractability, often too complex to be implemented in real-world real-time basis.

In this paper we analyze the behavior of a population of stochastic producers in electricity markets focusing on the collective impact of individual actions as they manifest through day-ahead market offers on an energy dispatch model consisting of a day-ahead and a realtime market. We draw inspiration from the El Farol Bar Problem (EFBP) [7], a specific example of a class of game-theoretic problems known as Minority Games [8], to study the market competition among self-interested participants. We employ a set of adaptive learning rules, introduced in [9], that through a probabilistic evolutionary process allow stochastic producers to successfully co-ordinate their offers under limited knowledge of the market conditions. We use a pool system based on IEEE96 [10] to show numerically that stochastic producers can derive simple adaptive strategies that converge dynamically to a steady state without relying on extensive records of generation profiles or market prices.

The rest of the paper is organized as follows: In Section II we provide and overview of the Minority Game and the El Farol Bar problem, while in Section III we provide the formulation that allows to study an electricity market as a Minority Game. In Section IV we illustrate the convergence properties on a simulated system, and we conclude in Section V. 


\section{Minority Games, Market Competition And} A BAR

In this section we describe how Minority Games can be used to study population dynamics in financial markets. Then we introduce key aspects of the El Farol Bar Problem, a specific case of a Minority Game.

\section{A. Minority Games}

In a Minority Game, a population of agents has to decide between options $\mathrm{A}$ and $\mathrm{B}$ with those belonging on the minority group by the end of the game considered as the winners. Naturally, the fact that those belonging in the minority group derive more benefits is very appealing in financial markets, given that market complexity is summarized under the famous mantra: 'Sell when everybody is buying and buy when everybody is selling'. This link is strongly represented in related literature [8], [11], [12] which generally considers a market that consists of $N$ participants who without loss of generality can either 'buy' or 'sell' a commodity at each time step $t$. For participant $i$, its action set is denoted by $a_{i}=\{-1,1\}$ where 1 corresponds to an action and -1 to its opposite.

At each evolutionary step $t$ of the MG, the gain (or loss), is equal to $\rho_{i}(t)=-a_{i} g(A(t))$, where $g($.$) a$ function of $A(t)=\sum_{i=1}^{N} a(t)$ a global measure that describes the state of the market at time $t$. For the choice of $g(A(t))$, [8] propose the sign function

$$
\operatorname{sgn}(A)=\left\{\begin{array}{rll}
-1 & \text { if } & A<0 \\
1 & \text { if } A>0 \\
0 & \text { if } A=0
\end{array}\right.
$$

while [11] propose $g(A(t))=A(t) / N$ as more appropriate for analytical mathematical calculations.

Based on the definition of the MG, $A(t)=0$ is the 'comfort threshold' as $A(t)<0$ suggests that the minority has chosen action 1 and for $A(t)>0$ the minority has chosen -1 . The intuition behind the choice of the sign function becomes clear as those who belong in the minority group are those with $a_{i}=-\operatorname{sgn}(A(t))$ and receive $|A(t)|$, while those in the majority have chosen $a_{i}=\operatorname{sgn}(A(t))$ and face a loss of $-|A(t)|$. Clearly, the gain of a market participant at each iteration of the game does not solely depend on the individual's actions; instead, it takes into consideration the global state the system as it aggregates all participants' actions.

\section{B. The El Farol Bar Problem}

The EFBP is a MG with a fixed threshold on the majority of the population at $60 \%$ as opposed to the MG with a threshold $50 \%+1$. It was defined by W. B. Arthur, inspired by the El Farol bar he frequented in Santa Fe [7]. The bar was popular on an Irish music night but Arthur felt uncomfortable when he perceived the bar to be overcrowded. Assuming a total population of $N=100$ he set the threshold at $\mathcal{N}=60$ and simulated the bar's attendance deriving predictors from the history of prior actions. The EFBP is considered a perfect example of 'bounded rationality', a departure from classical economic models assuming pure logic and 'deductive rationality'. Bounded rationality is arguably appropriate for modeling population dynamics in complex systems such as markets whereby individuals are aware only of their own valuations and their decision process is restricted by limits on the available information.

Therefore, the EFBP provides a realistic framework for modeling some of the complex operations in power systems. In particular, supply and demand in a microgrid utilizing generation from renewable and conventional sources and storage is modeled as a 'Potluck Game', a generalization of the EFBP with varying threshold levels [13]. Similarly, [14] achieve lower costs and increased stability, by deploying a MG based energy management system for smart buildings in a micro-grids.

\section{Inductive vs Adaptive Learning}

A common characteristic of MG based studies of populations in financial markets, power systems and in the El Farol bar, is the 'inductive learning' approach that requires that agents decide on their actions by predicting the global state of the system. They do so, by inferring the actions of everybody else, through a combination of rational rules and empirical evidence. Bell et al. in [9] provide an overview of the inductive learning approach, from simple deterministic methods (e.g. moving averages) to advanced methods involving genetic algorithms and neural networks. However, they argue that inductive learning emphasizes on the prediction of the state of the system at equilibrium without considering the evolution process towards that equilibrium, while having access to the distribution of the outcome.

Instead, they introduce 'adaptive learning', a process that allows members of a population to adapt the probability of undertaking a specific action, solely based on the history of their own experiences. As the probability slightly increases when an agent has a positive experience (e.g., an uncrowded bar) and slightly decreases after a negative experience (e.g., a crowded bar), the proposed adaptive learning rules are based on the principle that self-interested agents prefer to repeat enjoyable experiences and minimize the unpleasant ones.

\section{Adaptive Probabilistic LEarning Rules FOR PARTICIPATING IN ELECTRICITY MARKETS}

In this section we analyze the behavior of a popula- 
tion of stochastic producers in an electricity market that consists of a day-ahead and real-time market under the one-price balancing system, widely used in the USA and in Europe (incl. the UK, Germany, Benelux and Austria). We introduce a set of inductive and adaptive learning rules depending on stochastic producers' full or partial access on information about the outcomes of the dayahead and real-time markets.

\section{A. The Electricity Market as a Minority Game}

The 'market mantra' that links financial markets with Minority Games also applies on electricity markets under the one-price system where the optimal offer of a riskneutral price-taker stochastic producer is equal to to zero if the balancing price is higher than the day-ahead price, or equal to its capacity if the balancing price is lower than the day-ahead price. In a one-price balancing market the market price, $\lambda^{B}$, is higher than the day-ahead market price, $\lambda^{D}$, when the system is in up-regulation i.e. there is a deficit in power production due to deviations from day-ahead market offers. On the contrary, the balancing market price is lower than the day-ahead market price when the system is in down-regulation i.e. there is a surplus in power production.

Let $E^{*}$ be the upper limit a stochastic producer faces in its output, as it is defined by the technical specifications of the deployed wind farm units and let the real-time generation be equal to $E^{*} \widetilde{E}$, where $\widetilde{E} \in[0,1]$ is a realization of a random variable $Y$ which models the producer's stochastic output. The payment a producer receives under the one-price balancing market is

$$
P=\lambda^{D} E^{*} E^{D}+\lambda^{B} E^{*} \widetilde{E}^{B}
$$

replacing $\widetilde{E}^{B}=\widetilde{E}-E^{D}$ leads to an expression where $E^{D}$ is the only decision variable

$$
P=\left(\lambda^{D}-\lambda^{B}\right) E^{*} E^{D}+\lambda^{B} E^{*} \widetilde{E}
$$

with the variable $Y$ following a distribution $G$ defined by a set of parameters $\theta$ s.t. $Y \sim G(\widetilde{E} ; \theta)$.

Under Eq. (3) it is trivial to determine the optimal offer for a price-taker stochastic producer. That is, if $\lambda^{B}>\lambda^{D}$, it is in its best interest to offer zero power in the day-ahead market; while if $\lambda^{B}<\lambda^{D}$ it is in its best interest to offer its capacity $E^{*}$ on the day-ahead market. However, it is unrealistic to consider that only a single producer will determine and follow the optimal strategy that will maximize its revenues and all others will be merely observing without undertaking any action.

Furthermore, even if the assumption of the stochastic producers being price-takers and not able to exercise market power individually, is maintained, they can most
Minority Majority

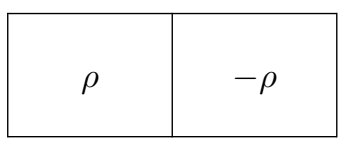

Fig. 1. Gains and losses in a minority game modeled electricity market.

certainly do so collectively. For example, if strategic stochastic producers' models predict a lower price in the balancing market, their optimal strategy will be to offer their nominal capacities at the day-ahead market. If the majority follows that strategy, the over-supply of cheap energy on the day-ahead market suggests that the market may be cleared at a relative low price. Consequently, having the majority of stochastic producers offer their capacities, will cause a deficit in the balancing market, given that a producer's output cannot exceed its nominal capacity. In turn, this may push up the balancing prices.

The observation that it is not optimal for a producer to offer its nominal capacity if all others also follow this strategy, relates to the behavior of a market population, whereby it is in an individual's best interest to act against the majority. The action set $a_{i}$ of stochastic producer $i$ facing the dilemma of either offering its nominal capacity or a zero bid, is denoted by $a_{i}=\{1,-1\}$ with 1 corresponding to an offer equal to its capacity and -1 to an offer equal to zero KWh. Fig. 1 summarizes the gains and losses for a producer at each time step in the evolution of the MG. Specifically, the gain is equal to $\rho_{i}=\operatorname{sgn}(A) \cdot E^{D}$ where $A=\sum_{i=1}^{N} a_{i}$, with a stochastic producer in the minority group receiving $\rho$, while one belonging in the majority group facing a loss equal to $-\rho$. This shows that stochastic producers decide on their actions based only on whether their action will put them in the minority or the majority.

We manipulate Eq. (3) so that stochastic producers' revenues express gains at each time step, by subtracting $\lambda^{B} E^{*} \widetilde{E}$, and then dividing by $E^{*}$.

$$
P^{*}=\frac{P-\lambda^{B} E^{*} \widetilde{E}}{E^{*}}=\left(\lambda^{D}-\lambda^{B}\right) E^{D}
$$

By doing so, the scaled revenue depends only on the day-ahead offer and the difference between the dayahead and real-time markets which dictates the producer's optimal strategy. It can be seen that $\rho=\mathrm{A} \cdot\left|P^{*}\right|$ with $E^{D}$ being equal to either $E^{*}$ or 0 .

\section{B. Learning Rules for Stochastic Producers}

Having modified the payment structure in the oneprice electricity market so that it can express the gains for stochastic producers at each iteration of the MG, we 


$$
p_{i}(t+1)= \begin{cases}0 & \text { if } p_{i}(t)-\epsilon(N(t)-\mathcal{N}) a_{i}(t)<0 \\ 1 & \text { if } p_{i}(t)-\epsilon(N(t)-\mathcal{N}) a_{i}(t)>1 \\ p_{i}(t)-\epsilon(N(t)-\mathcal{N}) a_{i}(t) & \text { otherwise }\end{cases}
$$

$$
p_{i}(t+1)= \begin{cases}0 & \text { if } p_{i}(t)-\epsilon \operatorname{sgn}(N(t)-\mathcal{N}) a_{i}(t)<0 \\ 1 & \text { if } p_{i}(t)-\epsilon \operatorname{sgn}(N(t)-\mathcal{N}) a_{i}(t)>1 \\ p_{i}(t)-\epsilon \operatorname{sgn}(N(t)-\mathcal{N}) a_{i}(t) & \text { otherwise }\end{cases}
$$

$$
p_{i}(t+1)= \begin{cases}0 & \text { if } p_{i}(t)-\epsilon(N(t)-\mathcal{N})<0 \\ 1 & \text { if } p_{i}(t)-\epsilon(N(t)-\mathcal{N})>1 \\ p_{i}(t)-\epsilon(N(t)-\mathcal{N}) a_{i}(t) & \text { otherwise }\end{cases}
$$

proceed to develop the adaptive learning rule that defines their interactions within the market. As mentioned in Section II-C adaptive learning rules are based on the simple intuition that market participants will tend to maximize their positive experience, in this case associated with belonging in the minority group. Based on [9] we depart from the classic literature in Minority Games [8] and the El Farol Bar Problem [7] relying on inductive learning approaches that assume market participants' capacity to predict the market outcome i.e. day-ahead and real-time prices and all other producers' production. In doing so, we provide an efficient explanation of market dynamics that does not rely on information that is either impossible or too costly to obtain.

Against this background, we introduce $p_{i}$ as the probability of stochastic producer $i$ offering its nominal capacity. Initially, we assume that there is $p_{i}(0) \%$ chance that a producer will offer its capacity with $p_{i}(0) \sim U(0,1)$. Let $\{1,0\}$ denote the actions available to a producer, $M$ their total number and $\mathcal{N}$ the threshold that defines majority and minority in a market. In consistence with MG literature, we assume that $M$ is an odd number $2 k+1$ with $k \in \mathbb{N}$ and $\mathcal{N}=k+1$. The value of $\mathcal{N}$ suggests that the cardinality of the minority set is equal to $k$ and of the majority set equal to $k+1$.

In this context, we assume that in the $t$-th iteration of the algorithm, each producer's action $a_{i}(t)$ is a random variable drawn from a Bernoulli random distribution with $p=p_{i}(t)$. Furthermore, $N(t)$ denotes the number of producers reporting their nominal capacities, with $N(t)=\sum_{i=1}^{M} a_{i}(t)$. The operation of the algorithm is simple: At each time $t$ the producer flips a biased coin with probability $p_{i}(t)$, with $p_{i}(t)$ increasing proportionally to $N(t)-\mathcal{N}$ on a positive outcome, and decreasing on a negative outcome. By positive and negative outcome we refer to the producer offering its nominal capacity ending in the minority or majority groups respectively.
Over time, producers gather information about the state of the market, which they 'store' in $p_{i}$, with the evolution of $p_{i}(t)$ described in Eqs. (5)-(7). It should be noted that the probabilistic element $a_{i}(t)$ in Eqs. (5) and (6) represents the adaptive learning process, while its absence in Eq. (7) suggests that producers have access to full information regarding the market's state, mimicking the information structure of the inductive learning process. Indeed, the rule in Eq. (7) is updated based on the total participation of the market at a previous time step, while rules in Eqs. (5) and (6) allow producers to decide based only on their prior experience.

Irrespective of how the learning rule is updated, there is a significant constraint given that $p_{i}(t)$ denotes a probability and therefore takes values in $[0,1]$. This may not necessarily be the case for $(N(t)-\mathcal{N}) a_{i}(t)$ or $N(t)-\mathcal{N}$, hence we introduce a parameter $\epsilon$ that defines how producers adapt to new information. Most importantly, parameter $\epsilon$ can be used to express the evolution of $p_{i}(t)$ based on a payoff scheme. Specifically, for the rule described in Eq. (6), the use of the sign function defined in Eq. (1), introduces a form of payoff similar to the gains or losses, in accordance to the payment structure in Fig. 1. A producer offering its nominal capacity expects to receive a gain equal to $\epsilon$ if it ends up in the minority group, or $-\epsilon$ if it ends in the majority group.

Consequently, we further manipulate $P^{*}$ to include $\epsilon$ by scaling Eq. (4) so it takes values in $[0,1]$. To do so, we introduce an upper bound for $\left|\lambda^{D}-\lambda^{B}\right|$ equal to the value of the lost load, denoted by $V_{L}$. The total revenue of a stochastic producer is given by

$$
P=V_{L} E^{*} P^{* *}+\lambda^{B} E^{*} \widetilde{E}
$$

where $P^{* *}$ is given by

$$
P^{* *}=\frac{\lambda^{D}-\lambda^{B}}{V_{L}} E^{D}
$$


which is then used as the basis of the parameter $\epsilon$ of the adaptive learning rules s.t.

$$
\epsilon=\frac{\left|\lambda^{D}-\lambda^{B}\right|}{V_{L}}
$$

To this end, the whole process is the following:

Step 0 The parameter of the Bernoulli trial that decides whether producer $i$ will offer its nominal capacity is initiated s.t. $p_{i}(t=0) \sim U(0,1)$

Step 1 All producers submit their offers in the dayahead market, with stochastic producers offering their nominal capacity with probability $p_{i}(t)$ and the market cleared at price $\lambda^{D}(t)$.

Step 2 Stochastic production is drawn from $G_{i}$ and balancing market is cleared at price $\lambda^{B}(t)$.

Step 3 The parameter $p_{i}(t+1)$ is updated based on $\epsilon$ from Eq. (10) and values of $\lambda^{D}(t)$ and $\lambda^{B}(t)$.

\section{NUMERicAl Simulations}

In this section, we illustrate the evolution of the producers' behavior in the market, through a numerical simulation based on a version of the IEEE Reliability Test System 1996 [10]. While we omit the network structure, we improve the setup by including marginal costs for conventional generation based on [15] and by adding flexible generators capable of providing up and down regulation following [16]. Finally, the cost of upregulation is assumed to be $10 \%$ higher than the dayahead cost and the cost of down-regulation $9 \%$ less than the day-ahead ahead costs ${ }^{1}$.

Regarding stochastic generation, we assume zero marginal cost [17] and cost free spilling action, while load shedding, $V_{L}$, induces a cost of $1000 £ / \mathrm{MWh}$. We guarantee feasibility of the market clearing by assuming that the total demand is at $80 \%$ of the conventional generation [16], while the total capacity of the 39 stochastic producers is at $30 \%$ of the demand. Stochastic generation uncertainty is modeled by a Beta distribution in consistence with the related literature (cf. [18], [17]) defined by mean $\mu_{i}$ and variance equal to 0.05 . The mean can be viewed as the point forecasts that the stochastic producers may submit as offers in the day-ahead market and for the simulations are sampled from a Uniform distribution $\mathrm{U}(0.3,0.6)$. The parameters $\alpha_{i}$ and $\beta_{i}$ of the Beta distribution are defined as

$$
\alpha=\frac{(1-\mu) \mu^{2}}{\sigma^{2}}-\mu, \quad \beta=\frac{(1-\mu) a}{\mu}
$$

We study the competition within the stochastic producers in a simulated market environment as it evolves

${ }^{1}$ The full dataset can be found on
http://dx.doi.org/10.5281/zenodo.56362

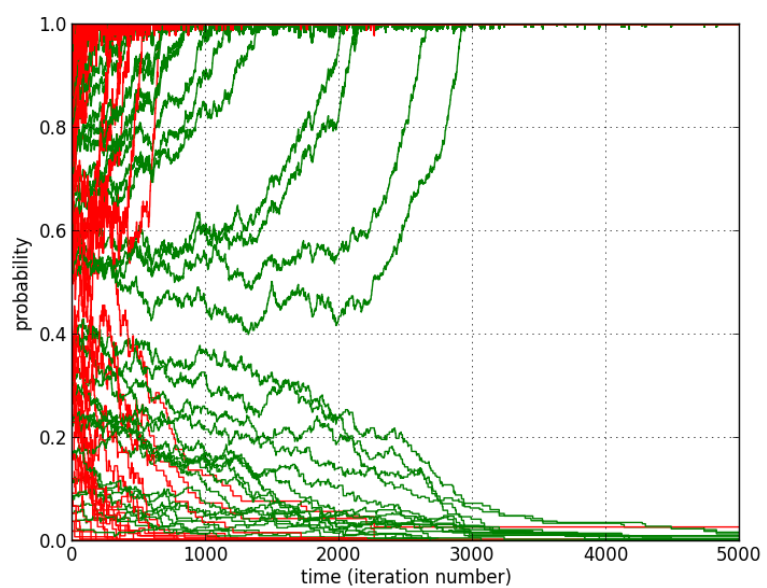

Fig. 2. Evolution of adaptive learning rules.

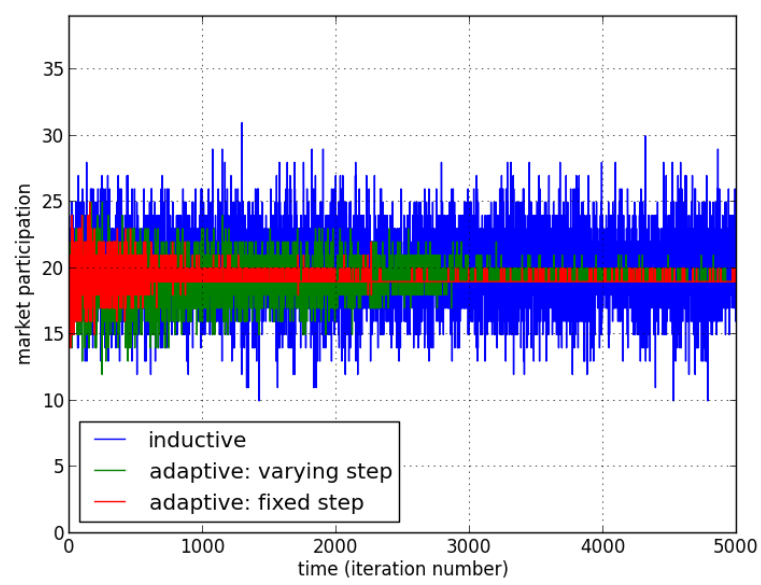

Fig. 3. Participation in the electricity market.

through 5000 time steps. In this context, Fig. 2 illustrates the convergence properties of the adaptive learning rules while Fig. 3 the evolution of the day-ahead market participation for all three learning rules. The 'adaptive: varying step' uses the $\epsilon$ derived from Eq. 10, while 'fixed step' assumes $\epsilon=0.01$. In Table I we provide some summary statistics for the three learning rules and a conventional setup where stochastic producers report their point forecasts in the day-ahead market.

Now, Fig. 2 shows that for a fixed step, the day-ahead market converges relatively fast to a state where the population is divided in two groups: those in the minority offering their nominal capacity and those in the majority bidding zero, while for the varying step the convergence is slower, due to the values of $\epsilon$ being close to 0.001 . This shows that producers manage to coordinate their behavior despite acting with little information available 
TABLE I

TOTAL PROFIT FOR WIND POWER PRODUCERS.

\begin{tabular}{ccccc}
\hline & varying & fixed & inductive & pt. forecast \\
\hline Avg. DA & 29572 & 29672 & 30958 & 25762 \\
Avg. RT & -4629 & -4664 & -6670 & -216 \\
Avg. total & 24943 & 25008 & 24287 & 25546 \\
Sd. Error & 38.81 & 38.54 & 42.49 & 31.11 \\
Eq. total & 26507 & 26507 & 26751 & n/a
\end{tabular}

and only interested in maximizing their own gains. As mentioned in [9], employing adaptive learning rules allows the market to achieve a Pareto efficient outcome, converging to a pure strategy Nash equilibrium.

Such behavior is an inherent property of the adaptive rules as opposed to the inductive learning one. As shown in Fig.3, the use of an inductive learning rule a pattern similar to the agents' attendance in the El Farol Bar as also mentioned in [9], [7]. The apparent noise in the market outcome is also captured by the standard deviation over the 5000 time steps, equal to $0.64,1.31$, 2.88 for the fixed, varying and inductive learning rules respectively. Finally, Table I shows that for MG based studies producers have increased profits in equilibrium (Eg. Total) compared to a market whereby all stochastic producers report their point forecasts in the day-ahead market (Avg. total for pt. forecast).

\section{CONCLusions AND Future Work}

In this paper we draw analogies between the El Farol Bar Problem, a specific example of a Minority Game and electricity markets. We study the competition among stochastic producers using a set of learning rules to identify their actions in the day-ahead market followed by a one-price balancing market. We illustrate through numerical simulations that adaptive learning rules can lead to the coordination of self-interested producers having access to minimum information on the outcome of the market and their peers' actions.

For future research we intend to generalize to both one-price and two-price balancing markets, while introducing additional uncertainty in the form of probabilistic estimates of market clearing prices. Furthermore we intend to focus on local aspects of the power system e.g., congestion issues between zones in wider geographical areas and develop more advanced learning rules that will be able to capture more complex behavior patterns that may arise e.g., incentive based learning for producers offering their full capacity in congested nodes.

\section{ACKNOWLEDGMENT}

The authors are partly supported by the Danish Council for Strategic Research (DSF) through the project ' $5 \mathrm{~s}$ -
Future Electricity Markets', No. 12-132636/DSF. Acknowledgments are due to Christos Ordoudis for feedback on the design of the system used in the simulations.

\section{REFERENCES}

[1] P. Pinson, C. Chevallier, and N. Kariniotakis, "Trading wind generation from short-term probabilistic forecasts." IEEE Transactions on Power Systems, vol. 22, no. 3, pp. 1148-1156, 2007.

[2] T. Jonsson, P. Pinson, and H. Madsen, "On the market impact of wind energy forecasts." Energy Economics, vol. 32, no. 2, pp. 313-320, 2010.

[3] M. Zugno, J. M. Morales, P. Pinson, and H. Madsen, "Pool strategy of a price-maker wind power producer." IEEE Transactions on Power Systems, vol. 28, no. 3, pp. 3440-3450, 2013.

[4] L. Barringo and A. Conejo, "Strategic offering for a wind power producer." IEEE Transactions on Power Systems, vol. 28, no. 4, pp. 4645-4654, 2013.

[5] S. Delikaraoglou, A. Papakonstantinou, C. Ordoudis, and P. Pinson, "Price-maker wind power producer participating in a joint day-ahead and real-time market." in Proceedings of 12th IEEE International Conference European Energy Market, 2015.

[6] S. A. Gabriel, A. Conejo, J. Fuller, B. Hobbs, and C. Ruiz, Complementarity Modeling in Energy Markets, ser. International Series in Operations Research \& Management Science. Springer, 2012, vol. 180

[7] W. B. Arthur, "Inductive reasoning and bounded rationality: The El Farol problem." American Economic Association, vol. 84, no. 2, pp. 406-411, 1994.

[8] D. Challet, M. Marsili, and Y.-C. Zhang, "Modeling market mechanism with minority game," Physica A: Statistical Mechanics and its Applications, vol. 276, no. 1-2, pp. 284-315, 2000.

[9] A. M. Bell, W. A. Sethares, and J. A. Bucklew, "Coordination failure as a source of congestion in information networks." IEEE Transactions on Signal Processing, vol. 51, no. 3, pp. 875-884, 2003.

[10] IEEE RTS Task Force of APM Subcommittee, "The ieee reliability test system-1996." IEEE Transactions on Power Systems, vol. 14, no. 3, pp. 1010-1020, 1999.

[11] E. Moro, Advances in Condensed Matter and Statistical Physics. Nova Science Publishers Inc., 2004, ch. Minority game: an introductory guide.

[12] T. Galla, G. Mosseti, and Y.-C. Zhang, Anomolous Fluctuation Phenomena in Complex Systems: Plasmas, Fluids and Financial Market. Research Signpost, 2008, ch. Anomalous fluctuations in Minority Games and related multi-agent models of financial markets.

[13] I. Maity and S. Rao, "Simulation and pricing mechanism analysis of a solar-powered electrical microgrid." IEEE Systems Journal, vol. 4, no. 3, pp. 275-284, 2010.

[14] C. Zhang, W. Wu, H. Huang, and H. Yu, "Fair energy resource allocation by minority game algorithm for smart buildings," in Design, Automation Test in Europe Conference Exhibition (DATE), 2012, 2012, pp. 63-68.

[15] D. Kirschen. Unit commitment data for modernized ieee rts-96. Accessed: 10-03-2016. [Online]. Available: http://www.ee.washington.edu/research/real/library.html

[16] A. J. Conejo, M. Carrión, and J. M. Morales, Decision Making Under Uncertainty in Electricity Markets. Springer, 2010.

[17] J. M. Morales, M. Zugno, S. Pineda, and P. Pinson, "Electricity market clearing with improved scheduling of stochastic production," European Journal of Operational Research, vol. 235, no. 3, pp. 765-774, 2014.

[18] X. Liu, "Impact of beta-distributed wind power on economic load dispatch," Electric Power Components and Systems, vol. 39, pp. 768-779, 2011. 